\title{
A mechanistic scale-up approach to the prediction of the geochemical evolution of sulfidic waste rock
}

\author{
DAVID WILSON ${ }^{1 *}$ RICHARD T. AMOS ${ }^{2}$ \\ JEFF B. LANGMAN ${ }^{3}$ LESLIE SMITH ${ }^{4}$ DAVID C. SEGO ${ }^{5}$ \\ DAVID W. BLOWES ${ }^{1}$ \\ ${ }^{1}$ Department of Earth and Environmental Sciences, \\ University of Waterloo, Waterloo, Canada \\ (*dwilson2@uwaterloo.ca) \\ ${ }^{2}$ Department of Earth Sciences, Carleton University, Ottawa, \\ Canada \\ ${ }^{3}$ Department of Geological Sciences, University of Idaho, \\ Moscow, USA \\ ${ }^{4}$ Department of Earth, Ocean and Atmospheric Sciences, \\ University of British Columbia, Vancouver, Canada \\ ${ }^{5}$ Department of Civil and Environmental Engineering, \\ University of Alberta, Edmonton, Canada
}

Prediction of the geochemical evolution of mine-waste rock is of critical importance because of the potential impacts of mining wastes on a local environment. Prediction is often conducted using the results of small-scale kinetic tests in conjunction with empirically derived scale factors in a process commonly referred to as scale-up. One of the overall goals of the Diavik Waste Rock Project was the development of a mechanistic scale-up approach for the prediction of the geochemistry of mine-waste effluent. The project included laboratory and field experiments to investigate the geochemical evolution of waste rock at different scales. The laboratory study consisted of humidity cell $(0.1 \mathrm{~m})$ experiments; the field studies consisted of medium-scale (2 $\mathrm{m})$ lysimeter experiments and large-scale test pile $(15 \mathrm{~m})$ experiments. A conceptual model of the geochemical evolution of the waste rock was implemented at the humidity cell scale using the multicomponent reactive transport code MIN3P. Mass- and temperature-dependent rate parameters for reactions involving sulfide content, host mineralogy, and secondary minerals were calibrated by simulating the effluent geochemistry of the humidity cell experiments. The resulting calibrated model was then implemented for the field-scale experiments using site measured temperature and infiltration parameters that provided conditions representative of the experiments. Results of the simulations at the three experiment scales indicated that the geochemical evolution of the field experiments was captured well by the scaled conceptual model. These simulations showed that a reactive transport model calibrated using results of humidity cell experiments has the potential to predict the geochemical evolution of mine-waste rock at the large scale without use of empirical scaling factors. 